\title{
Interkulturalität als Projekt
}

\section{Dieter Heimböckel / Manfred Weinberg}

Die nachfolgenden Ausführungen unternehmen den Versuch einer Fortschreibung. Sie greifen Gedanken zur Interkulturalitätsforschung auf, die einerseits aus dem Sonderforschungsbereich Literatur und Anthropologie Ende der $1990 \mathrm{or}-$ Jahre an der Universität Konstanz hervorgegangen und andererseits im Rahmen dieser Zeitschrift kürzlich als Vor-Überlegungen zur Interkulturalität veröffentlicht worden sind (vgl. Rieger/Schahadat/Weinberg 1999 sowie Heimböckel 2013a). Für die konkreten Literaturbeispiele bildet das aktuelle Themenheft Übersetzen. Praktiken kulturellen Transfers am Beispiel Prags den thematischen Bezugsrahmen. Dabei versteht sich die Fortschreibung selbst als Teil des Projektes, um das es in dem vorliegenden Beitrag hauptsächlich geht.

\section{JeNSEITS DES ÜBLICHSEINS}

Wer auf Reisen geht, sucht in der Regel nach Orientierung. So ergeht es auch Karl Roßman, als er sich in Franz Kafkas Roman Der Verschollene auf den Weg nach Amerika macht. Es ist aber alles andere als eine freiwillige Reise, die der junge, von einem Dienstmädchen verführte und von seinen Eltern daraufhin verstoßene Mann in die neue Welt unternimmt. Es ist, dessen ist sich auch Karl bewusst, vor allem eine Reise ohne Rückfahrschein, sodass er sich umso gewissenhafter auf das Kommende vorbereitet. Er liest viel über das Land am anderen Ende des Atlantiks, vermutlich mehr, als er lesen müsste. Aber das teilt er mit seinem literarischen Ziehvater, der, vor und zeitgleich mit der Abfassung des Romans, wie ein Schreibtischethnologe ausführliche landeskundliche Studien betreibt. Vielleicht haben Karl Roßmann und Franz Kafka Arthur Holitschers gerade eben erschienene Reisebeschreibung Amerika heute und morgen (Holitscher 1912) sogar gemeinsam gelesen. Vieles scheint dafür zu sprechen, denn als Roßmann gleich zu Beginn des Romans in den Hafen von New York einfährt, erblickt er »die schon längst beobachtete Statue der Freiheitsgöttin wie in einem plötzlich stärker gewordenen Sonnenlicht.« (Kafka 2008: 9) Doch statt der Fackel hält sie ein Schwert in ihrer Hand - als »Symbol der erbarmungslosen Gewalt« (Hermsdorf 1961: 69) und Hinweis auf den bevorstehenden Kampf in einem durch und durch technisierten und ökonomisierten Gesellschaftssystem, wie manche Interpreten meinen (vgl. Nicolai 1981: 52). Eindeutiger wäre der Zusammenhang wohl gewesen, wenn Kafka die ersatzlose Streichung einer 
Textvariante rückgängig gemacht und es bei der abschließenden Formulierung der Eingangsszene belassen hätte. Denn ursprünglich sollte es an dieser Stelle heißen: »Er [Roßmann] sah zu ihr auf und verwarf das über sie Gelernte.« (Kafka 2002a: 123)

Aber um Eindeutigkeit geht es in Kafkas Der Verschollene nicht. Es geht vielmehr um Verstehen, und zwar um ein Verstehen, das von Anfang als verschoben zu denken ist. Verschiebungen sind dabei keine Richtigstellungen, noch führen sie dazu. Im Akt der Verschiebung wird das Verstehen vielmehr aufs Spiel gesetzt. Sie bildet die Voraussetzung dafür, dass Grenzen (des Verstehens - und nicht nur diese) überschritten und Grenzziehungen, in denen sich ein gewohnheitsmäßiges bzw. übliches Denken äußert, reflektiert und zugleich außer Kraft gesetzt werden. ${ }^{1}$ So bezieht Karl zwar wie Kafka sein Wissen aus »Gelernte[m]«, aber der Roman macht keinen Hehl daraus und stellt es immer wieder zur Disposition. Gleich im ersten Kapitel, in der Begegnung mit dem Heizer, steht die Annäherung an die Außenwelt ganz im Zeichen der eurozentrisch-stereotypen Vor-Urteile Karls: ») Sind Sie ein Deutscher?< suchte sich Karl zu versichern, da er viel von den Gefahren gehört hatte, welche besonders von Irländern den Neuankömmlingen in Amerika drohen.« (Kafka 2008: 10) Die vorgängige Aneignung lenkt Karls Wahrnehmung der amerikanischen Welt, und diese bestimmt sich aus dem, was der eurozentrische Blick ihm eingibt. »[A]uf Mitleid durfte man hier nicht hoffen und es war ganz richtig, was Karl in dieser Hinsicht über Amerika gelesen hatte; nur die Glücklichen schienen hier ihr Glück zwischen den unbekümmerten Gesichtern ihrer Umgebung wahrhaft zu genießen.« (Ebd.: 45) So bleibt er stets Europäer, der gleichsam immer wieder aufs Neue in Amerika ankommt und dazu gezwungen wird, das Wahrgenommene mit den Bildern, die seiner Einstellung zugrunde liegen, abzugleichen. »>Da gibt es also auch schon in Amerika alte Häuser<, sagte Karl. >Natürlich , sagte Klara lachend und zog ihn weiter. >Sie haben merkwürdige Begriffe von Amerika.< >Sie sollen mich nicht auslachen<, sagte er ärgerlich. Schließlich kannte er schon Europa und Amerika, sie aber nur Amerika.« (Ebd.: 71)

Karl kennt Amerika - aber er kennt Amerika wie jemand, der von Anfang an seine Kenntnisse unter einen Vorbehalt gestellt sieht. Sein »Da gibt es also auch schon in Amerika alte Häuser« ist Ausdruck dieses Vorbehalts - und dieser Vorbehalt manifestiert sich als Staunen. Karl ist noch jung, er hat das Leben noch vor sich und wird so das eine oder andere Mal von dem, was da auf ihn zukommt, überrascht - vielleicht häufiger sogar, als ihm lieb ist. Denn insofern sich im Staunen »eine grundlegende Verunsicherung und semantische Leere« vor dem Unvertrauten zeigt (Schnyder 2013: 95f.), wirft es ihn aus der Bahn seines gewohnten bzw. angeeigneten Wissens. Das Staunen unterbricht - und bricht aufgrund der Plötzlichkeit seiner Wucht in ihn ein. Karl geht so, bei aller Breite und Tiefe des Kontinents, den er mit seiner Einfahrt in den Hafen von

1 | Zur Verschiebung (oder Deplatzierung) als einem Verfahren, das das Ungewohnte im Gewohnten aufscheinen lässt und geläufige Aneignungen, Wahrnehmungen und Begriffe in der Schwebe hält, vgl. auch Heimböckel 2013b: 36. 
New York betritt, beständig wie auf einer Grenze, die ihm das Staunen als liminales Phänomen eingibt. Dabei ist das Staunen auch bei ihm ein Moment, in dem er sich zwar der Begrenztheit seines Wissens bewusst wird, in dem sich aber auf der anderen Seite sein Blick für etwas Neues öffnet. In der Erkenntnis der eigenen Begrenztheit entsteht so auch die Möglichkeit, die Grenze zu überschreiten.

Im Rahmen von Interkulturalität spielt das Staunen als eine »Weise bewußtwerdenden Nichtwissens « (Guzzoni 2012: 12) heuristisch eher eine marginale Rolle - zu Unrecht, wie wir meinen. Denn es löst den Beobachter aus den Fesseln seiner Kulturbedingtheit und ermöglicht einen auf absolute Wertmaßstäbe verzichtenden Kulturvergleich, indem es nicht nur »die Selbstverständlichkeit eigener kultureller Erfahrungen durchbricht« (Schlesier 1996: 155), sondern auch interkulturelle Grundlagenkategorien wie das Eigene und das Fremde selbst in Frage stellt. Insofern übernimmt das Staunen in der interkulturellen Konstellation gewissermaßen eine doppelte Funktion: und zwar die des Vehikels ebenso wie die der Irritation. Damit ist gleichzeitig ein Verständnis von Interkulturalität verknüpft, das sich aus bestimmten - begrifflichen, definitorischen etc. - Vorgaben zu lösen sucht und das Prozesshafte in den Vordergrund rückt. Das hier zur Diskussion stehende Verständnis von Interkulturalität ist, worauf zunächst einzugehen sein wird, nicht dasjenige einer »Disziplin« (Yousefi/ Braun 2011: 27) oder eines Paradigmas (vgl. Schmitz 1991). Denn anders als das Paradigma, »das gleichzeitig Bollwerk gegen veraltetes wie neues Nichtwissen « ist (Gugerli/Sarasin 2009: 8), liefert die perspektivische Offenheit des Projekts erst die Möglichkeit, sie, d.h. Interkulturalität, im Kontext von Grenzen einerseits und Nichtwissen und Staunen andererseits zu reflektieren.

\section{Der Projektgedanke}

Zusammengesetzt aus lateinisch >pro < und >iacere < (werfen) und abgeleitet aus dem lateinischen Partizip >proiectus<, ist im Begriff >Projekt< die Bedeutung >vorwärts-, vorwerfen, hervortreten lassen, hin-, niederwerfen< aufgehoben. Dabei versteht man bereits seit dem 18. Jahrhundert unter einem Projekt ein Konzept bzw. einen Entwurf, im engeren Sinne ein Vorhaben und den Plan dazu. Bei Projekten geht es schon in dieser Zeit um »die planmäßig begrenzte Unternehmung, welche neue Problemlösungen, deren Durchführbarkeit und Wünschbarkeit, Probleme und Folgeprobleme erkundet.« (Stanitzek 1987: 138) Nach der heutigen DIN-Norm 69901 des Deutschen Instituts für Normung gilt ein Projekt als Vorhaben, »das im Wesentlichen durch die Einmaligkeit der Bedingungen in ihrer Gesamtheit« gekennzeichnet ist, wie zum Beispiel »(1) Zielvorgabe, (2) zeitliche, finanzielle oder andere Begrenzungen«, und das sich durch Abgrenzung gegenüber anderen Vorhaben und eine projektspezifische Organisation auszeichnet (Lindner 2010: 43). Wenn hier vom Projekt der Interkulturalität gesprochen wird, ist damit tendenziell allerdings ein anderer Projekt-Begriff intendiert. Darauf wird gleich wieder zurückzukommen sein, doch 
muss aus heuristischen Gründen erst noch die bislang entfaltete Vorstellung dessen, was heute prinzipiell unter einem Projekt verstanden wird, weiterverfolgt werden.

In Erweiterung dieses Verständnisses gilt eine Aufgabenstellung in der Regel dann als Projekt, wenn »das zu lösende Problem relativ komplex erscheint, der Lösungsweg zunächst unbekannt ist, eine Zielrichtung und ein Zeitrahmen vorliegen und/oder bereichs-/fachübergreifende Zusammenarbeit erforderlich ist «. ${ }^{2}$ Für den Projektbegriff, auf den wir abzielen, ist das Unbekannte seines Ausgangs ebenso wie die sich zum Teil widersprechenden Teilziele konstitutiv. Der wesentliche Unterschied ergibt sich allerdings vor allem aus der zeitlichen Entgrenzung, indem - analog etwa zum »Projekt der Moderne«, das von Jürgen Habermas ausgerufen wurde (vgl. Habermas 1981) - das Prozessuale in den Vordergrund gerückt wird. Ein Projekt in diesem Sinne kann zwar einen ungefähren Ausgangspunkt benennen, die Voraussetzungen, unter denen sich der Gedanke des Projekts formierte; das Ergebnis ist aber nach Maßgabe seines Prozesscharakters ganz und gar offen. Damit kehren wir zu den neuzeitlichen Ursprüngen des Projekts und seines Machens zurück, in das 17. Jahrhundert, als das Projektemachen, angesiedelt zwischen barocker Spekulationslust, alchemistischem Laborieren, spektakulärem Erfindertum auf der einen Seite und einer systematischen Förderung sozialer, technischer und administrativer Innovationen auf der anderen Seite, förmlich Mode wurde und einen jener Schauplätze des Wissens markierte, »an dem Realität und Illusion, das Machbare und das Denkbare, das Wahrscheinliche und das Unmögliche beständig miteinander in Konflikt « gerieten (Lazardzig 2007: 247). Gehört zum Projekt im Sinne der DIN-Norm, dass es auf ein Gelingen ausgerichtet und so am Ende (und mit dem zeitlich eingeplanten Ende) seines Status als Projekt enthoben ist, wofür es betriebswirtschaftlich geschulte Projektmanager und institutionell beflissene Facilitators (sog. Kommunikationslotsen) gibt, so ist für das Projekt im klassischen Sinne die »Möglichkeit des Nichtzustandekommens, des Scheiterns, des Aufgebens immer mit im Visier; sonst handelt es sich nicht um Projekte« (Stanitzek 1987: 138). Es geht hier aber nicht um die Frage, ob bei dem, was wir heute gewohnheitsmäßig als Projekt bezeichnen, so etwas wie eine Entzauberung des ursprünglichen Projektdenkens vorliegt, sondern inwiefern, bezogen auf das Phänomen der Interkulturalität, an diesen Projektgedanken angeschlossen werden kann. Ein Anschluss ließe sich etwa mit Alexander Kluge herstellen, der davon ausgeht, dass Projekte im Grunde Vorgriffe und Ausbrüche in eine nicht übersehbare Ferne sind (vgl. Kluge 2006: 14). Denn diese Ferne lässt sich inhaltlich, aber auch zeitlich für das Projekt der Interkulturalität in Anschlag bringen - und auch dafür, dass es Projektion eines aus der Not oder Krise gesetzten Anfangs in etwas Unbekanntes ist.

So war mit dem Begriff der Interkulturalität seit den 1970er-Jahren die Funktion verknüpft, politisch realisierbare Konzepte für Konfliktlösungen in der >Nation of Immigrants < zu erarbeiten (vgl. Schnell 2000: 231); gleichzeitig

2 | Online unter: http://de.wikipedia.org/wiki/Projekt [Stand: 31.10.2014]. 
bediente sie, verbreitet durch die praktische Disziplin der interkulturellen Kommunikation, »einen Wissens- und Kompetenzbedarf für internationale Beziehungen nicht nur, aber primär geschäftlicher Art« (Mecklenburg 2008: 91). Ihr weiterer Erfolgsweg zu einem Leitbegriff und vielbeachteten Forschungsthema steht in einem engen Zusammenhang mit der Etablierung der >Cultural Studies < und >Intercultural Studies < in Amerika und ihrer britischen und kontinental-europäischen Rezeption in den 1970er- und 8oer-Jahren (vgl. Hess-Lüttich 2003: 76). Im Zuge der Forderung nach Dialog und kritischem Konsens in einer globalisierten Welt und angesichts des Umstandes, dass Interkulturalität sich in den 1980er-Jahren sukzessive als leitende und disziplinübergreifende Forschungskategorie etabliert hat, hat sie sich bald schon den Ruf eingehandelt, der »Rabattlösung « modischer Studieninteressen zu dienen (Wierlacher 2003: 16). Bezeichnenderweise stammen solche und ähnliche Anwürfe mehrheitlich aus dem Munde ihrer leidenschaftlichsten Apologeten.

Dass der lnterkulturalitätsforschung der gesinnungswissenschaftliche Ruf vorauseilt, wohlgemeint, aber konzeptionell unzureichend zu sein (vgl. FornetBetancourt 2007: 121), und dass wir gar »nicht genau« wissen, »was Interkulturalität eigentlich ist bzw. sein soll«, wie es noch jüngst in einem Grundlagenartikel zur Black Box >Interkulturalität< schlagwortartig formuliert worden ist (Földes 2009: 504), hat aber unter anderem damit zu tun, dass aufgrund der Involviertheit unterschiedlicher Fakultäten und der Vielstimmigkeit im gesellschaftlichen Diskurs nicht nur eine Vielzahl von Ansätzen und Lösungswegen kursieren. Die mitwirkenden Organisationen und Instanzen gehorchen auch unterschiedlichen Sachlogiken, die sich nur in seltenen Fällen auf den Begriff bringen lassen. Interkulturalität ist insofern weit davon entfernt, ein Paradigma zu sein, und weist stattdessen Eigentümlichkeiten auf, die für ein Projekt spezifisch sind. Darum lässt sie sich auch nicht so einfach - wie eine überkommene Lehrmeinung - verabschieden, so als wäre über sie das letzte Wort bereits gesprochen und damit ihr Scheitern ausgemacht. ${ }^{3}$ Ihr Scheitern ist auch deshalb nicht ausgemacht, weil in diesem Projekt das Scheitern nicht ein Resultat, sondern selbst schon im Denken an und über Grenzen angelegt ist. Dem liegt ein Verständnis von Interkulturalität zugrunde, das sich als Methode zur Umgestaltung bestehender Denk- und Handlungsformen versteht und das sich darauf einlässt, landläufige Selbstverständlichkeiten und Sehgewohnheiten zu hinterfragen. Interkulturalität geht insofern einher mit dem kulturanthropolo-

3 | Vgl. zuletzt Kimmich/Schahadat 2012: "Im Zeitalter von Globalisierung und Migration ist die Vorstellung von einer zugleich an ein Volk, eine Nation und an einen spezifischen Ort gebundenen Kultur im Herderschen Sinne offenbar obsolet geworden. Man versucht vielmehr, die Fluidität, die Dynamik und die Grenzüberschreitungen zwischen Kulturen zu begreifen. Ein Begriff, der auf diese Situation reagiert, ist der der Transkulturalität. Als kulturwissenschaftliches Paradigma löst er sowohl das Konzept der Interkulturalität als auch die Prämissen der Post Colonial Studies der 1990er Jahre ab." (7) Zur Problematik einer Interkulturalitätskritik, die sich, wie in diesem Fall, an Wolfgang Welsch und dessen Verständnis von Transkulturalität orientiert, siehe die Ausführungen unter Punkt 3 des vorliegenden Beitrags. 
gischen Ausbruch aus dem, was Alfred Schütz einmal als »Denken-wie-üblich« bezeichnet hat (Schütz 1972: 58). Im Denken-wie-üblich ist das Fremde das aufgefasste Andere. Es ist wie das Eigene eine Setzung, dessen das Denken-wieüblich bedarf, damit es sein Üblichsein bewahrt. Interkulturalität hinterfragt daher Repräsentationen des Eigenen und Fremden nicht - sie hätte ansonsten einen Begriff davon -, sondern setzt deren Dekonstruktion voraus. Dazu gehört auch, dass sie das mit dem Eigenen und Fremden vermittelte Wissen in Frage stellt. Das, was gilt und was nicht gilt (denn dies ist das durch die Geltung Ausgeschlossene), übersetzt sie in eine Sprache, die dem Denken-wie-üblich unvertraut ist. Interkulturalität ist Übersetzung in eine unvertraute Sprache - und das heißt in eine Sprache, die davon absieht weiterzuwissen.

\section{Die Grenzen der Interkulturalität}

Wenn, wie hier vorgeschlagen, das Projekt der Interkulturalität auf einer Verschiebung beruht, die eine >Überschreitung von Grenzen< ebenso meint wie die >Reflexion und das Außer-Kraft-Setzen von voraus-gesetzten Grenzziehungen<, wenn zudem das das interkulturelle Projekt prägende Staunen ein >auf der Grenze Gehen ‘ und damit ein >liminales Phänomen` vorstellt, dann beginnt (und endet) die Frage nach (einem angemessenen Verständnis von) Interkulturalität mit der Frage nach dem Status und der besonderen Ausprägung kultureller Grenzen. Im Projekt der Interkulturalität drängt sich also die Frage nach den Grenzen der Interkulturalität (genitivus subjectivus) auf. Vor dem Hintergrund des schon Gesagten kann es sich dabei aber nur um nicht eindeutige und nicht fixe, sondern immer schon verschobene und weiterhin jederzeit verschiebbare Grenzen handeln. Nur mit einem solchen Verständnis der Grenze(n) lässt es sich vermeiden, die Grenzen der Interkulturalität (räumlich wie zeitlich) auch als genitivus objectivus denken zu müssen.

Ein zu harmloses Verständnis kultureller Grenzen hat vor allem Wolfgang Welsch den Konzepten der Interkulturalität vorgeworfen, gegen die er den vermeintlich angemesseneren Begriff der Transkulturalität ins Spiel brachte. In einem programmatischen Aufsatz Welschs unter dem Titel Was ist eigentlich Transkulturalität? liest man:

Die begriffliche Revision, die das Konzept der Transkulturalität vorschlägt, bezieht sich [...] vor allem [...] auf die extensionale Bedeutungsdimension von 'Kultur. Es rät, diese Extension anders zu verstehen als traditionell. Nämlich nicht mehr nach dem alten Modell klar voneinander abgegrenzter Kulturen, sondern nach dem Modell von Durchdringungen und Verflechtungen. Und zwar deshalb, weil Kultur heute - so die Behauptung de facto derart permeativ und nicht separatistisch verfasst ist. (Welsch 2012: 26) ${ }^{4}$

Später heißt es:

4 | Hierbei handelt es sich um die gekürzte Fassung von Welsch 2009. 
Zeitgenössische Kulturen sind extrem stark miteinander verflochten. Die Lebensformen enden nicht mehr an den Grenzen der Einzelkulturen von einst (der angeblichen Nationalkulturen), sondern überschreiten diese, finden sich ebenso in anderen Kulturen. Die Lebensform eines Ökonomen, eines Wissenschaftlers oder eines Journalisten ist nicht mehr einfach deutsch oder französisch, sondern - wenn schon - europäisch oder global geprägt.

Und intern sind zeitgenössische Kulturen weithin durch Hybridisierung gekennzeichnet. Für jedes Land sind die kulturellen Gehalte anderer Länder tendenziell zu Binnengehalten geworden. (Ebd.: 28)

Wie man sieht, orientiert Wolfgang Welsch sein neues Konzept der Transkulturalität deutlich an einer zeitlichen Entwicklung: In einem für ihn überkommenen Kulturmodell, das er am Ende des 18. Jahrhunderts maßgeblich durch Johann Gottfried Herder geprägt und vertreten sieht, seien Kulturen als Kugeln verstanden worden - und zu diesem Kugelmodell gehörten »ein internes Homogenitätsgebot und ein externes Abgrenzungsgebot« (ebd.: 27). So wird - bei aller Globalisierung und Pluralisierung von Kultur im späten 20. und frühen 21. Jahrhundert (aber erst seitdem?) - offensichtlich, dass Welsch die Komplexität vorangegangener Kulturmodelle und eines früheren In-, Gegen- und Miteinanders von Kulturen vorsätzlich und sträflich unterschätzt. Schon Herders Kultur-Konzept lässt sich der jüngeren Forschung zufolge nicht einseitig auf eine Tendenz zur organizistisch-ganzheitlichen Prägung festlegen. ${ }^{5}$ Wer demgegenüber dem emphatisch empfohlenen Konzept der Transkulturalität einen zeitlichen Index mit auf den Weg gibt, muss unterstellen, dass sich frühere Kulturen tatsächlich zu den zugeschriebenen >harmloseren< Bedingungen der Interkulturalität begegnet sein könnten. Wir halten es dagegen mit der zeitlich nicht indizierten Bestimmung Jean-Luc Nancys: »Jede Kultur ist in sich >multikulturell<, nicht nur, weil es immer eine vorgängige Akkulturation gegeben hat und es keine einfache und reine Herkunft gibt, sondern vor allem deshalb, weil der Gestus der Kultur einer des Vermischens ist.« (Nancy 1993: 6f.) Wohlgemerkt: alle Kulturen und immer schon!

Der zeitliche Index, unter dessen Voraus-Setzung Welsch sein Konzept der Transkulturalität legitimiert hat (und das Konzept der Interkulturalität radikal delegitimieren wollte), hatte übrigens zur höchst negativen Konsequenz, dass in unzähligen und nicht enden wollenden Diskussionen auf vor allem germanistischen Fach-Tagungen die Frage nach Inter- und Transkulturalität immer wieder nach einer Logik des >noch nicht< vs. >nun aber < verhandelt und damit jedem Anspruch einer an und für sich tragfähigen Modellierung der zur Rede stehenden Phänomene abgeschworen wurde. Zudem vergrundsätzlichen sich solche Debatten allermeist irgendwann, sehen dann von der bei Welsch vorausgesetzten historischen Diagnose ab und werden zum völlig unfruchtbaren >Glaubenskriegく, dem Welsch durch eine seinerseitige Fundamentalisierung allerdings durchaus >vorgearbeitet< hat: In diesem werfen die Anhänger der

5 | Vgl. Gaier 2007 sowie den differenzierten Forschungsüberblick bei Dembeck 2010. 
Transkulturalität den Anhängern der Interkulturalität vor, an begrenzenden (und somit in ihrer Analysekraft begrenzten) Kulturmodellen festzuhalten und umgekehrt lautet der Vorwurf meist, dass Transkulturalität viel zu sehr auf Hybridität als grenzenlose Vermischung setze und damit jede Einheit(lichkeit) zu Unrecht negiere. Demgegenüber gilt jedoch:

Die Debatte um ınter-ı und Iranskulturalität ist zuletzt so unproduktiv wie die vergleichbare um ,Inter-ı und 'Transdisziplinaritätı. Im Grunde wissen alle an der Diskussion Beteiligten, welch komplexe Formen des kulturellen Austauschs zu denken sind Von daher sind weniger die Diagnosen Welschs unangemessen, als vielmehr die von inm so massiv vorgetragene These, all das lasse sich im Rahmen der Interkulturalität nicht denken. (Langenohl/Poole/Weinberg 2015)

Ein auch nur oberflächlicher Blick auf in den Wissenschaften diskutierte GrenzKonzepte zeigt, dass man entweder den für die Frage nach dem In-, Mit- und Gegeneinander von Kulturen zu eindeutigen Grenzbegriff des Nationalstaats findet (im Englischen unter dem Begriff >border <) oder (im Englischen unter dem Stichwort >boundary $<$ mit einer langen Liste von verschiedenen GrenzBegriffen aus ganz unterschiedlichen Disziplinen, u.a. Psychologie, Soziologie, Mathematik, Physik, konfrontiert wird. ${ }^{6}$ Dabei ist das Verständnis der Grenzen nach dem Modell von >border für die Frage nach der Grenze als Leitmodell interkultureller Raumkonzepte offensichtlich viel zu eng gefasst (und zu Recht die eigentliche Bezugsgröße von Welschs Kritik), während die Angebote unter dem Stichwort >boundary< deutlich zu vielfältig und kaum auf interkulturelle Situationen anwendbar sind.

Es lässt sich an dieser Stelle auf Karl Roßmann zurückkommen. Denn dieser rekurriert, in den unendlichen Weiten Amerikas angekommen, in erstaunlicher Weise auf seine >Heimat $>$. Diese wird zunächst als $>$ Eigenes $<$ herangezogen, von dem her sich das >Fremde < verstehen (oder zumindest in seiner Andersartigkeit beschreiben) lässt:

[H]ier wanderten keine einzelnen Marktweiber zur Stadt, wie in Karls Heimat, aber doch erschienen hie und da große flache Automobile, auf denen an zwanzig Frauen mit Rückenkörben, also doch vielleicht Marktweiber, standen und die Hälse streckten, um den Verkehr zu überblicken und sich Hoffnung auf raschere Fahrt zu holen. (Kafka 2008: 110f.)

Der Diagnose der klaren Differenz zwischen Europa (resp. Deutschland) und Amerika folgt hier prägnant die Mutmaßung der Ähnlichkeit (»also doch vielleicht Marktweiber«), was das ebenso komplexe wie problematische Zusam-

6 | Vgl. etwa die entsprechenden Wikipedia-Artikel: "border" (online unter: http:// en.wikipedia.org/wiki/Border [Stand: 31.10.2014]) und "boundary" (online unter: http://en.wikipedia.org/wiki/Boundary [Stand: 31.10.2014]).

7 | Vgl. zur Verwendung des Begriffs der Heimat seitens der Autoren der Prager deutschen Literatur Weinberg 2014. 
menspiel von erinnertem Eigenen und wahrgenommenem Fremden aufruft. Später heißt es:

Vieles erinnerte Karl an seine Heimat und er wußte nicht, ob er gut daran tue, New-York zu verlassen und in das Innere des Landes zu gehn. In New-York war das Meer und zu jeder Zeit die Möglichkeit der Rückkehr in die Heimat. Und so blieb er stehn und sagte zu seinen beiden Begleitern, er habe doch wieder Lust, in New York zu bleiben. (Ebd.:

Die Reise ins Innere des Landes wird hier als Aufbruch in die vergleichslose Fremde imaginiert; schließlich hat sich Roßmann auf die amerikanische Provinz auch nicht lesend vorbereitet. Als Delamarche ihm zuredet, New York den Rücken zu kehren, ihn, wie es wörtlich heißt, »einfach weitertreiben wollte, ließ er sich nicht treiben und sagte, daß er doch wohl noch das Recht habe über sich zu entscheiden.« Seine beiden Bekannten erklären ihm sodann,

daß Butterford viel schöner als Newyork sei und beide mußten inn noch sehr bitten, ehe er wieder weiter gieng. Und selbst dann wäre er noch nicht gegangen, wenn er sich nicht gesagt hätte, daß es für inn vielleicht besser sei, an einen Ort zu kommen, wo die Möglichkeit der Rückkehr in die Heimat keine so leichte sei. (Ebd.)

Abgesehen von der Ambivalenz des doppelten >Gehens < (einmal das Weitergehen im Gespräch, zum anderen der Gang in die Fremde) steht hier der Status des Fremden/der Fremde selbst in Frage, wobei diese Infragestellung an die Möglichkeit zur Rückkehr in die Heimat gebunden wird, die unschwer als Frage nach dem Sinn der Bezugnahme auf sie interpretierbar ist.

Bemerkenswert ist dabei auch die Profilierung der so aufgerufenen Heimat an einer anderen Stelle. Über ein Gespräch mit der Köchin heißt es:

"Entschuldigen Sie bitte", sagte er, "daß ich mich noch gar nicht vorgestellt habe, ich heiße Karl Roßmann." "Sie sind Deutscher, nicht wahr?" "Ja", sagte Karl, "ich bin noch nicht lange in Amerika." "Von wo sind Sie denn?" "Aus Prag in Böhmen", sagte Karl. "Sehn Sie einmal an", rief die Oberköchin in einem stark englisch betonten Deutsch und hob fast die Arme, "dann sind wir ja Landsleute, ich heiße Grete Mitzelbach und bin aus Wien. Und Prag kenne ich ja ausgezeichnet, ich war ja ein halbes Jahr in der Goldenen Gans auf dem Wenzelsplatz angestellt." (Ebd.: 134) ${ }^{8}$

Karl wird als Deutscher identifiziert, was angesichts des nachgeschobenen Herkunftsorts »Prag in Böhmen« unangemessen ist, da jenes Prag zur Entstehungszeit des Romans nicht in >Deutschland <, sondern in >Kakanien $<$, also Österreich-Ungarn lag (womit man das »Deutscher« eigentlich als >Deutschsprachiger $\mathrm{zu}$ verstehen hat). Zudem wird mit Böhmen ein ganz anderes, weil regionales Erklärungsmuster der Heimat aufgerufen. Neben der Sprache

8 In der Nachfrage der Köchin, ob er Deutscher sei, wiederholt sich Roßmanns Frage an den Heizer. 
figuriert also ein (falsches) nationales sowie ein regionales Verständnis von Heimat - und eine im Vergleich zu Amerika ganz andere kulturelle Ordnung, die eine des Austauschs und der Wanderung über Europa prägende kulturelle Grenzen hinweg ist: Die Wiener Köchin Mitzelbach hat einige Zeit in Prag gearbeitet.

Diese Mehrfachkodierungen kann man für die Ambivalenz auch der Heimat (jedenfalls von Amerika aus betrachtet) stehen lassen, womit deutlich wird, dass >Heimat< im Roman Der Verschollene eben nicht als homogene Einheit (eines heimelig Vertrauten und somit Fraglosen) verstanden wird. Dies zeigt sich auch, wenn man sich die Beschreibung jener Szene genauer anschaut, die zu Roßmanns >Verschickung< nach Amerika den Anlass gab, die Verführung durch das Dienstmädchen: Sie »drückte ihren nackten Bauch an seinen Leib, suchte mit der Hand, so widerlich daß Karl Kopf und Hals aus den Kissen heraus schüttelte, zwischen seinen Beinen, stieß dann den Bauch einigemale gegen ihn, ihm war als sei sie ein Teil seiner selbst und vielleicht aus diesem Grunde hatte ihn eine entsetzliche Hilfsbedürftigkeit ergriffen. Weinend kam er endlich nach vielen Wiedersehenswünschen ihrerseits in sein Bett.« (Ebd.: 36 [Hervorh. d. Verf.]) Dies ist nichts anderes als die Erzählung einer Auflösung von (Körper-) Grenzen im sexuellen Akt, die in einer Einrichtung von nun räumlichen Grenzen durch seine >Verschickung über den Atlantik wieder aufgerichtet werden sollen. Doch gerade die Rückkehr zu einer die Grenzen wieder stabilisierenden Ordnung gelingt ja in Amerika nicht; vielmehr beginnt das beschriebene Spiel der stets neuen (und nie >aufgehenden $<$ ) Korrelierung von Eigenem und Fremdem, das man - im Sinne dieses Aufsatzes - auf das Projekt der Interkulturalität (und seinen spezifischen Umgang mit Grenzen) beziehen kann. Dass dieses Spiel nur als >Prozess $<$ (um nicht >Process $<$ zu schreiben) verstanden werden sollte, zeigt eine letzte Erwähnung von Heimat im Verschollenen. Karls >Freunde $<$ können es nicht begreifen,

daß Karl über zwei Monate in New York gewesen war und kaum etwas anderes von der Stadt gesehen hatte, als eine Straße. Und sie versprachen inm, wenn sie in Butterford genug verdient hätten, mit inm nach New York zu gehn und inm alles Sehenswerte zu zeigen und ganz besonders natürlich jene Örtlichkeiten, wo man sich bis zum Seligwerden unterhielt. Und Robinson begann im Anschluß daran mit vollem Mund ein Lied zu singen, das Delamarche mit Händeklatschen begleitete, das Karl als eine Operettenmelodie aus seiner Heimat erkannte die inm hier mit einem englischen Text viel besser gefiel, als sie inm je zuhause gefallen hatte. (Ebd.: 114)

Nach dem imaginierten Gang in die gänzlich unverständliche Fremde wird hier also eine sich daran anschließende Rückkehr nach New York vorgestellt, das Karl aber ja gar nicht umfassend, sondern nur höchst bruchstückhaft, also begrenzt kennengelernt hat - und jene unbekannten Orte des »Seligwerden[s]《 in New York werden mit einer Operettenmelodie illustriert, die Karl als >heimatliche< identifiziert, die ihm aber nun in ihrer >Übersetzung < in die Fremde (und fremde Sprache) sogar besser gefällt. So wird das dichotomische Spiel mit 
dem Eigenen und Fremden außer Kraft gesetzt, was als erster Schritt in der prozesshaften Etablierung eines > Dritten < oder besser > Liminalen $<$ mit durchaus offenem Zukunftshorizont erscheint.

\section{INTERKULtURALItät 1999 REVISITED}

Ausdrücklich an das Konzept der Interkulturalität anschließend, erschien 1999 in der Publikationsreihe des Konstanzer Sonderforschungsbereichs Literatur und Anthropologie ein von Stefan Rieger, Schamma Schahadat und Manfred Weinberg herausgegebener Sammelband unter dem Titel Interkulturalität. Zwischen Inszenierung und Archiv. Im Vorwort wird Interkulturalität gleich zu Beginn mit der »rethnologische[n] oder anthropologische[n] Wende< der Literaturwissenschaft « (Rieger/Schahadat/Weinberg 1999: 10) verbunden und in den Horizont von deren kulturwissenschaftlicher Öffnung verschoben. Vor allem aber wird der Begriff der Kultur pluralisiert, wie es zum theoretischen >Grundbestand < dieses SFBs gehörte. So werde jedoch der »Kulturbegriff selbst unscharf [...], weshalb das im Begriff der Interkulturalität implizierte Verständnis von Kultur notwendigerweise eines ist, das sich unablässig mit den Fragen von Kohärenz, Homogenität und Diskretheit konfrontiert sieht« (ebd.). Dieser unscharfe Kulturbegriff wird allerdings von den Herausgebern nicht als Manko verstanden, sondern sozusagen als Aufgabe, mit den Worten dieses Aufsatzes: als Projekt. Martin Heidegger hat hinsichtlich etwa der kantischen Form einer Wesensanthropologie formuliert: »Anthropologie ist jene Deutung des Menschen, die im Grunde schon weiß, was der Mensch ist und daher nie fragen kann, wer er sei.« (Heidegger 1980: 109) Dem lässt sich begegnen, indem man nicht mehr nach dem »Menschen als Menschen « (und von daher eben nach seinem Wesen) fragt, sondern »nach ihm als Ägypterin, Buddhisten oder Sprecherin des Türkischen« (Rieger/Schahadat/Weinberg 1999: 11). Doch entgeht auch eine solche Verschiebung zu einer kulturanthropologischen Perspektivierung der bloßen Bestätigung der eigenen Voraussetzungen nicht; ihre Chance liegt aber darin, sich zu diesen Voraussetzungen selbstreflexiv verhalten zu können und somit in der Erforschung von Kulturen die Infragestellungen, die der Plural von Kulturen mit sich bringt, unablässig zu bedenken.

Auf dieser Grundlage wagen sich die Herausgeber in ihrem Vorwort an eine Definition (ihres Verständnisses) des Interkulturalitätsbegriffs:

Kulturen (und mit innen Interkulturalität) kann es nur geben, wenn sich ein Punkt angeben läßt, an dem die eine Kultur aufhört und die nächste anfängt. Solche Differenzierbarkeit von Kulturen erhält im topographischen Modell der Grenze ihre allegorische Zuschreibung. Doch auch wenn diese Grenze die Bedingung der Möglichkeit von Interkulturalität bildet, heißt dies nicht, daß interkulturelle Begegnungen an und auf dieser Grenze stattfinden. Die Begegnung zweier Kulturen findet vielmehr statt, wenn eine Kultur in das abgegrenzte Terrain der anderen einbricht. Interkulturalität setzt somit beides voraus: eine Grenze zwischen den Kulturen - und ihre Überschreitung. (Ebd.) 
Hier wird - und zwar als Bestimmung der Interkulturalität - jedenfalls nicht auf ein Kugelmodell von Kulturen, die immer nur >zusammenstoßen<, sich aber niemals durchdringen können, referiert. Auf das im Konzept der Interkulturalität aufgerufene »Zwischen « fokussierend, heißt dies aber, dass es einerseits nicht die Zwischenstellung der Grenze selbst meinen kann, anderseits aber auch nicht unabhängig von der Grenze/von Grenzen zu denken ist. Das wird im Vorwort im Ausgriff auf Jurij Lotman und den Begriff der Hybride von Michail M. Bachtin weiter entwickelt, was an dieser Stelle nicht noch einmal aufzunehmen ist.

Im Vergleich zum hier vorgeschlagenen Projektgedanken von Interkulturalität scheint einem solchen Verständnis noch die Verzeitlichung zu fehlen. Doch findet sich diese in der Erläuterung des zweiten Teils des Band-Titels Zwischen Inszenierung und Archiv, der, wie die Verfasser ausführen, das herkömmlich eben zwischen den Kulturen angesiedelte und an eine Grenze gebundene »Inter« der Interkulturalität zu einem innerkulturellen verschiebt. Im Horizont ihrer eigenen Kultur begegnen die dieser Kultur Zugehörigen dem oder den Anderen und Unzugehörigen. >Zugehörigkeit < meint somit aber, dass eine solche Begegnung niemals unvorbereitet geschieht, sondern immer schon unter der Voraus-Setzung eines (vermeintlichen) Wissens um den Fremden (im Rahmen des Denkens-wie-üblich), auf das zurückgegriffen wird.

Doch auch wenn das Inventar dieser Bilder des (eigenen) kulturellen Gedächtnisses die Art und Weise präfiguriert, wie wir unsere Begegnung mit der anderen Kultur jeweils inszenieren, so determinieren sie diese nicht vollständig. Man kann aus den archivierten Verständnismustern zwar nicht einfach aussteigen, sie lassen sich aber durch konkrete Erfahrungen variieren, vielleicht präzisieren. Inszenierung und Archiv bilden auf diese Weise den performativen Rahmen für die Begegnung mit dem Anderen. Später ist im Vorwort von der jeweiligen »Neu-Inszenierung kultureller Bilder« (ebd.: 16) die Rede. Interkulturelle Begegnungen zeigen sich somit als Wiederholungen vergangener Begegnungen mit dem Anderen und Fremden, an die man sich erinnert, weil die von diesen früheren Begegnungen abgeleiteten Bilder zur Konstitution des durch den Anderen aktuell in Frage gestellten Eigenen gehören. Mehr noch: Der Andere nötigt mich zur Identifizierung mit der eigenen Kultur, die die Muster seiner Identifizierung (und Identität) als Fremder bereit hält. Nun gibt es jedoch kein identisches Wiederholen und kein vollständig adäquates Erinnern. »Wenn sich Kulturen aber nicht zu schließen vermögen, dann nistet ihn ihnen selbst ein Zwischen, das jener Ort sein könnte, an dem Interkulturalität den ihr zustehenden Platz findet.« (Vgl. ebd.)

Dass sich angesichts eines solchen Verständnisses von Interkulturalität die Rede von einem mit diesem vermeintlich stets verbundenen »Kugelmodell« und seinem »interne[n] Homogenitätsgebot « und »externe[n] Abgrenzungsgebot« von selbst erledigt, bedarf wohl keines Kommentars. Ebenso offensichtlich ist, in welcher Weise wir mit unserem Vorschlag, Interkulturalität als Projekt zu denken, daran anknüpfen. Allerdings verabschieden wir uns von der damals so vorsichtigen Rede eines bloßen »Moment[s] der Destabilisierung des Eigenen« 
und einer nur >kleinen Differenz< (vgl.: ebd.) in der erinnernden Wiederholung der Stereotype zum Anderen. Vielmehr >dramatisieren< wir diese Differenz nun: Wenn das Vorwort zum Sammelband Interkulturalität. Zwischen Inszenierung und Archiv immerhin schon die Statik eines Denkens von Interkulturalität (somit als Konzept oder Paradigma) hinter sich lässt und Veränderung konzeptuell einbezieht, so betonen wir nun noch deutlicher den grundsätzlichen Prozess-Charakter und den damit notwendigerweise mitzudenkenden offenen Ausgang eines solchen Prozesses oder eben genauer: Projekts.

Hinzuweisen ist übrigens noch darauf, dass manche Grundsatz-Annahmen des SFB Literatur und Anthropologie im aktuellen Konstanzer Exzellenzcluster Kulturelle Grundlagen von Integration ihre Fortschreibung erfahren haben. Ein für unsere Belange entscheidender Punkt ist dabei, dass in der Konzeptbildung dieses Exzellenzclusters Identitäten verflüssigt werden. Identität wird nicht mehr verstanden »als ein quasi natürlicher Dauerzustand des Selbstbewusstseins sozialer Akteure«, sondern als »Effekt einer Dramatisierung von Differenz.« Identitätsfragen stellen sich danach nur in kritischen Übergangsphasen und können in ruhigeren Zeiten latent bleiben. So aber wird Identität zur »situationsabhängige[n] Kategorie «.9 Auf eine solchermaßen situative und verflüssigte Identität wird in diesem Aufsatz am konkreten Beispiel Prags noch einmal zurückzukommen sein.

lnsofern also im Phänomen des lnterkulturellen ein Zwischenraum bezeichnet ist, in dem vermeintlich feste Grenzen verschwinden und neue Grenzen gezogen werden, steht der Kulturaustausch prinzipiell unter dem Vorzeichen der Gewissheitsreduktion. Es gibt zwar nach Bernhard Waldenfels, »keinen Ort jenseits der Kultur, der uns einen unbefangenen und unbeschränkten Überblick gestatten würde. Als Europäer können wir der eigenen Kultur so wenig entfliehen wie dem eigenen Leib und der eigenen Sprache.« (Waldenfels 2000: 247) Aber Waldenfels' Argument bezieht seinen Impuls aus einer transkulturalitätskritischen Position, die, xenopolitisch motiviert, den dritten Raum - besser spräche man vom liminalen Raum - in der fremdkulturellen Begegnung stark zu machen sucht. Der liminale Raum ist der eigentliche Ort nicht des Wissens, sondern des Nichtwissens als Gegenstand der lnterkulturalität. Zu einer so verstandenen lnterkulturalität liegen, weil sich hinter ihr (im Verständnis des Nichtwissens) »eine prinzipiell nicht aufhebbare Ungewissheit möglicher Ereignisse« verbirgt (Willke 2002: 11), Hegemonie- ebenso wie Alleinvertretungsansprüche naturgemäß quer. Die aus der Anlehnung an reflexive Theoriebildung hervorgehende Provokation führt dazu, Interkulturalität konsequenterweise im Modus des Nichtwissens zu denken. Die Infragestellung der Fremdperspektive und die Be- und Verarbeitung von Nichtwissen sind dabei lediglich zwei Seiten einer Medaille (vgl. Beck 1996: 314).

9 | Online unter: https://exzellenzcluster.uni-konstanz.de/157.html?\&L=wqdszfehuck aj [Stand: 31.10.2014]. 


\section{5. "SO HOCH» - Staunen alS BeWUSSTWERDENDES NichtWISSEN}

Im Sinne einer projektbezogenen Vorstellung von Interkulturalität ist die interkulturelle Aktivität ein Experiment und der Beobachter ein Experimentator. Er weiß nicht, was sich jenseits des Denkens-wie-üblich verbirgt. In diesem Nichtwissen generiert Interkulturalität ihr grenzüberschreitendes Potential.

In der Andersheit des Anderen stößt das Wissen an seine Grenzen. Denn wenn sie gewusst würde, wäre das Andere nicht mehr anders, sondern dem (eigenen) Wissen einverleibt. Das Andere (bzw. das aufgefasste Andere) steht daher bzw. aufgrund seiner »zugänglichen Unzugänglichkeit« (Waldenfels 1999: 42; weshalb die Rede über das Andere eine Rede auf der Grundlage einer Setzung des Anderen ist) unter dem Schutz des Nichtwissens. Umgekehrt geht am Wissen das Andere zunichte, weil es im Moment seines Wissens aufhört, seinen Anspruch auf Andersheit einzulösen. Es bringt das Andere zum Verschwinden, während es im Nichtwissen unangetastet bleibt. Im Nichtwissen über das Andere offenbart es seine eigentümliche Andersheit. Das Gewahrwerden der eigentümlichen Andersheit manifestiert sich als Staunen. Es ist ein Staunen einerseits über das Andere, andererseits über das Denken-wie-üblich und seine Begrenztheit. Das Staunen initiiert den Ausbruch aus dem Denkenwie-üblich und setzt damit ein Staunen über die Begriffe des Eigenen und Anderen frei.

Ein solches - anthropologisches - Staunen ist vom Staunen, das den Anfang der Philosophie bildet, freilich zu unterscheiden. Die Verwunderung, die Platon und Aristoteles als Ausgangspunkt des Philosophierens nahmen, indem sich die Menschen, wie es in der aristotelischen Metaphysik heißt, »anfangs über das nächstliegende Unerklärte verwunderten, dann allmählich fortschritten und auch über Größeres Fragen aufwarfen« (Aristoteles 1995: 6), läuft auf deren Beseitigung hinaus. Für die Philosophie als faktische Staunensentfernung ist das Staunen nicht Gewinn, sondern Mangel; Platon hatte es sogar mit unangenehmen Empfindungen wie Schwindel und Erstarren zusammengebracht, und die Pointe der Philosophie sollte gerade darin bestehen, »sich nicht auf das einzulassen, was schwindelig und erstarren macht«, sondern was imstande ist, sich zur Sphäre der Ideen zu erheben, »die kein Mehr und kein Weniger kennen, sondern fest und unveränderbar sind.« (Heinrich 2000: 30f.) Das Staunen selbst aber ist ein Verstoß gegen diese wie jede Form der Unveränderbarkeit, es bezeichnet für denjenigen, der staunt, einen Ausnahmezustand, mit dem er oder sie aus dem Verhältnis der Vertrautheit heraustritt und für einen Moment der Plötzlichkeit (oder länger) aus der gewohnten Bahn geworfen wird. Die Philosophie strebt danach, diesen Zustand aufzuheben. Ähnlich »wie Iris, die Tochter des Thaumas, als Götterbotin zwischen Erde und Himmel vermittelt und darin Eros gleicht, ist auch das Philosophieren ein Versuch, das Nichtwissen in ein Wissen zu überführen.« (Martens 2003: 45)

Bezogen auf Alteritätszusammenhänge führt ein solches Staunen dazu, dass in der Wissensüberführung der und/oder das Fremde aufhört, fremd zu sein. Schon Montaigne hat in Über die Gewohnheit und daß man ein überkomme- 
nes Gesetz nicht leichtfertig ändern sollte (De la coustume et de ne changer aisément une loy reçue) jedoch darauf hingewiesen, dass es sich bei der Fremdwahrnehmung um keine Einbahnstraße handelt, sondern um einen reziproken Prozess, bei dem, was jeweils Eigenes und Fremdes ist, letztendlich ununterscheidbar zusammenfällt:

Die Gewöhnung schläfert den Blick unseres Urteils ein. Die Barbaren setzen uns nicht stärker in Verwunderung, als wir sie, und mit nicht mehr Grund, wie ein jeder eingestehen würde, wenn ein jeder, nachdem er diese fremdartigen Beispiele durchwandert hätte, sich über die eigenen zu beugen und gesunderen Sinnes zu vergleichen wüßte. (Montaigne 1953: 159f.)

Renate Schlesier sieht in Montaignes Überlegungen zum Staunen den Anfang der Anthropologie verbürgt. Unser Staunen über Fremde, schreibt sie, sollte nicht »blindmachen für das Staunen der Fremden über uns, und wer dieses Staunen der Fremden nicht übersieht, lernt, auch über die eigene Kultur zu staunen.« (Schlesier 1996: 55) Über die eigene Kultur zu staunen, hieße, sich demjenigen, was in ihr fremd erscheint, auszusetzen, wodurch freilich das Eigene im Verhältnis zum Fremden nicht mehr different bzw. distinktiv erscheint. Das Eigene und das Fremde verlieren auf diese Weise nicht nur ihren Wert als kulturdistinktive Beziehungsgrößen, sie erweisen sich auch als Projektionen und Erfüllungsgehilfen der Verkennung im Prozess alteritärer Wahrnehmungsvorgänge.

In diesem Sinne schreibt Georg Christoph Lichtenberg in seinen Sudelbüchern: »Der Amerikaner, der den Kolumbus zuerst entdeckte, machte eine böse Entdeckung.« (Lichtenberg 1971: 166) Gabriele Brandstetter formuliert dazu in ihrer Baseler Antrittsvorlesung zur Literaturwissenschaft als Kulturpoetik von 1999:

Solche Umkehrung der europäischen Perspektive aus einer Politik der Entdeckungen, der kritische Blick auf die damit verbundenen Macht-Konfigurationen vermittelt eine folgenreiche und höchst moderne Erkenntnis: daß nämlich der Augen-Blick der Begegnung mit dem Fremden zwei Seiten hat. Der Amerikaner, der den Kolumbus entdeckt, blickt der europäischen Kultur ins Auge und spiegelt ihre eigene Befindlichkeit. Solche Begegnung, als dialogische, beruht auf der Wahrnehmung eines kulturellen Bruchs. In die Leerstellen des Unverständlichen, des Unlesbaren des Anderen schießen Diskursstrategien und Zeichenformationen ein, die das Unbegreifliche und Nicht-Formulierbare in die vertrauten Zeichen der eigenen Sprache und Zeichen-Konventionen übertragen sollen: ein Suchen nach Worten und Gesten, um das Trauma solcher Encounter-Situationen zu bewältigen. (Brandstetter 1999: 306f.)

Gehört nach Ute Guzzoni zum Erstaunen und Staunen »die Erfahrung sowohl eines eigenen Sich-fremd-Wissens wie der Fremdheit des Begegnenden « (Guzzoni 2012: 14), so muss im Rahmen dieser Erfahrung die kontextaufbrechende Wucht des Staunens und die dadurch initiierte Begriffsauflösung mit reflektiert 
werden. Im Außer-Ordentlichen des Staunens hätte demnach auch das Fremde keinen zuschreibbaren Ort.

Ein solches Staunen beschreibt keinen Mangel, sondern einen Gewinn. Und der Gewinn liegt im Bewusstwerden eines Nichtwissens, das das angeeignete bzw. gelernte Wissen überrascht. Man könnte mit Martin Seel vielleicht sogar von der Lust sprechen, »die ihre Erfüllung in einer Befriedigung ungeahnter Erwartungen findet.« (Seel 2009: 45) Nichtwissen ist in diesem Fall nicht die Kehr- oder dunkle Seite des Wissens, sondern ein Korrektiv zu der im Wissen angelegten Strategie der Vereinnahmung dessen, was gewusst werden soll, und zugleich der Modus, der auf Anerkennung des Nichtgewussten zielt.. ${ }^{10}$

Dass das Staunen naturgemäß nicht vom Staunenden losgelöst werden kann und auf ihn und seine Erfahrung zurückwirkt, ist dabei unzweifelhaft und erinnert an Uwe Timms Ausführungen zum Schreiben über fremde Welten und seine Vorstellung vom Staunen darüber, »wie die Menschen, wie die Dinge beschaffen sind, das heißt, anders sein können, als man selbst ist« (Timm 1997: 42). Gemeint ist damit zugleich ein Staunen, das von allem abweicht, was uns die Imagination zur Verfügung stellt. In den Worten des Ethnologen Fritz Kramers: Die anderen Gesellschaften verfügen »über Lebens- und Kunstformen, Weltbilder und Erfahrungsweisen [...], die wir nicht hätten erfinden können.« (Kramer 1977: 8 [Hervorh. im Orig.]) Insofern stellt das Staunen einen Verstoß gegen die Identifizierungstradition dar, die »projektive[n] Verkennung[en]« (Dietsche 1984: 5) Vorschub leistet und an deren Korrektur und/oder Außerkraftsetzung sich wiederum erweist, inwieweit sich Staunen einstellt bzw. ob es überhaupt zugelassen wird.

Man kann Kafkas Der Verschollene vor diesem Hintergrund als eine Art Kontrafaktur auf das Wissen bzw. auf den Mythos vom Wissen lesen. Zwar haben wir es bei Roßmann auch mit einer projektiven Verkennung zu tun, sie zeitigt bei ihm allerdings andere Konsequenzen. Er kannte Amerika aus Büchern und darüber hinaus so, wie man sich in der Antike eine Vorstellung von den im Westen, jenseits der Säulen des Herkules liegenden elysäischen Auen und Inseln der Seligen machte - Amerika, mit dessen Entdeckung die alte europäische Vision von dem Paradies auf Erden Wirklichkeit geworden zu sein schien, war schon vor Christoph Kolumbus ein erfundenes Land gewesen. Auf die Frage Christopher Newmans, ob sie etwas über den Genueser Weltensegler wisse, antwortet eine französische Kopistin in der Erstfassung von Henry James' Roman The American mit ebenso spitzfindiger wie abgründiger Ironie: »Bien sûr! He invented America; a very great man.« (James 1949: 6) Das erfundene Amerika bildet so die Folie der Inkongruenzen, die zwischen Karls Perspektive und der Neuen Welt an die Oberfläche treten. Das Wahrgenommene selbst verschiebt sich ins Phantastische und wird solchermaßen, wie im Falle der schwerttragenden Freiheitsstatue, ein zweites Mal erfunden.

10 In diesem Sinne sieht Ernst Tugendhat im Staunen einen Vorgang, bei dem sich das, was das Staunen hervorruft, dem Verstehen und damit der Erklärung entzieht (vgl. Tugendhat 2004: 159). 
Im Schwert, das sie trägt, kündigt sich das Unvertraute an, das sich im Roman zu einer verborgenen, insbesondere der Großstadtbeschreibung zugrundeliegenden Ästhetik des Erhabenen sublimiert. Karls erstauntes »So hoch« (Kafka 2008: 9) ist noch Zeichen der sprachlich kaum zu meisternden Überwältigung, die sich seiner beim Anblick des Wahrzeichens bemächtigt. Und je weiter er in das Land hineinzieht, desto breiter wird es, könnte man in Abwandlung eines Goethe-Wortes meinen (vgl. Goethe 1985: 105). Aber Karl müsste eine konkrete Vorstellung von dieser Breite gewinnen, er müsste sie ausfüllen mit Wissen und Erträgen seiner Wahrnehmungsernte. Stattdessen staunt er unentwegt und immer wieder. »Der Held ist in Amerika der exemplarische Fremde, der sich das weite Land mit ungläubigem Staunen erschließt, letzthin aber dabei scheitert, eine neue Heimat zu finden«, schreibt Peter-André Alt (2008: 356) in seiner Kafka-Biografie. Abgesehen davon, dass das Scheitern angesichts eines fragmentarisch gebliebenen Romans nicht das letzte Wort gewesen sein muss, liegt aber gerade im Staunen des Protagonisten die Unmöglichkeit, das Land zu erschließen, begründet. Zu einer Erschließung kommt es unter anderem deshalb nicht, weil das vorgängige Wissen der Erfahrung, die Karl macht, nicht standhält und weil die Unentwegtheit seines Versuches, einen festen Grund unter den Füßen zu bekommen, ebenso beharrlich einer Deplatzierung ausgesetzt wird. Die Konsequenz, mit der Karl unbeirrt seines Weges zieht, irritiert und beunruhigt dabei in dem Maße, in dem seine mit der angestrebten Akkulturation einhergehenden Bemühungen, das Unvertraute vertraut zu machen, ihn immer wieder mit neuen Szenarien des Unvertrauten konfrontiert. Bei Karl bildet so die projektive Verkennung die Grundlage für ein Staunen, mit dem das vorgängige Wissen in ein Nichtwissen überführt wird und das auch deswegen ein Nichtwissen bleibt, weil »das Wissen selbst kein Haben des Gewußten ist, keine Einvernahme, Assimilation oder sonstige Identifizierung (Guzzoni 2012: 13). Und es bleibt ein Nichtwissen, weil selbst das Gewusste sich nicht erschließt.

\section{Vom Horizont des Staunens}

Die vorstehenden Ausführungen sollten klar gemacht haben, dass der unsererseits vorausgesetzte Begriff des Staunens (anders als der an den Anfang der Philosophie gesetzte) nichts vorstellt, was zu überwinden ist. Gleichwohl findet er seine nächstliegende Anwendung selbstverständlich in der oben schon (mit Brandstetter) benannten >Encounter-Situation< einer ersten Begegnung. Das von uns gemeinte Staunen wird danach aber nicht von Worten und Gesten >überschrieben<, die das »Trauma solcher Encounter-Situationen zu bewältigen« helfen sollen, sondern persistiert - resp.: Selbst wenn es Aneignungsversuche in diesem Sinne gibt, >vergeht $<$ das Staunen durch sie nicht. Damit ist aber nahegelegt, dass man, um auf die oben angestellten Überlegungen zu den »Grenzen der Interkulturalität« zurückzukommen, einen angemessenen Grenzbegriff möglicherweise am besten in der Auseinandersetzung mit solchen Regionen findet, in denen verschiedene Kulturen schon Jahrhunderte 
lang zusammenleben, ihnen eigentlich also das Staunen übereinander schon lange vergangen sein sollte, was jedoch eine durchaus unangemessene Beschreibung wäre. Dabei handelt es sich jeweils um Kulturräume, die eben nicht von Staatsgrenzen zwischen den Kulturen geordnet wurden, sondern in denen sich zwei oder mehr Kulturen (in) einen Raum teilten, wie es eben auch für den Kulturraum der böhmischen Länder gilt, der nach der gängigen Formel von »Juden, Deutsche[n], Tschechen« (vgl. etwa Brod 1918) bewohnt war. Wir bleiben also für eine letzte Frage nach den Grenzen der Interkulturalität weiterhin bei den bisher schon herangezogenen Beispielen aus diesem Kulturraum.

Egon Erwin Kisch beschreibt die Interkulturalität des Prager Stadtraums zum Anfang des 20. Jahrhunderts wie folgt:

Mit der halben Million Tschechen der Stadt pflog der Deutsche keinen außergeschäftlichen Verkehr. Niemals zündete er sich mit einem Streichholz des Tschechischen Schulengründungsvereins eine Zigarre an, ebenso wenig ein Tscheche mit einem Streichholz aus einem Schächtelchen des Deutschen Schulvereins. Kein Deutscher erschien jemals im tschechischen Bürgerklub, kein Tscheche im Deutschen Casino. Selbst die Instrumentalkonzerte waren einsprachig, einsprachig die Schwimmanstalten, die Parks, die Spielplätze, die meisten Restaurants, Kaffeehäuser und Geschäfte. Korso der Tschechen war die Ferdinandstraße, Korso der Deutschen ,der Graben،

$[\ldots]$

Die deutsche und die tschechische Universität, die tschechische und die deutsche Technische Hochschule waren einander so fern, als wäre die eine am Nordpol, die andere am Südpol. (Kisch 1990: 78f.)

Kisch denkt somit die Stadt Prag im Zeichen streng voneinander abgegrenzter, jeweils eindeutig nationalkulturell kodierter Räume, anders gesagt: als Mosaik. Damit steht er im Übrigen nicht alleine. Von Pavel/Paul Eisner etwa stammt die Formel, dass die Autoren der Prager deutschen Literatur in einem dreifachen Ghetto gelebt hätten: als Deutsche unter Tschechen, als Juden unter Christen und als sozial Höhergestellte unter sozial niedriger Gestellten (vgl. Eisner 1948).

Ganz anders liest sich die Beschreibung des Prager Stadtraums bei Vilém Flusser, der 1920 als Sohn einer jüdischen Familie in Prag geboren wurde und in seiner 1992 erschienenen »philosophische[n] Autobiographie« Bodenlos schreibt:

Selbstredend, man war Prager, das stand nicht in Frage. Es war der Boden, auf dem sich alle anderen Fragen stellten. Aber war man als Prager Tscheche, Deutscher oder Jude? War man überhaupt berechtigt, die jüdische Dimension mit den beiden anderen auf dieselbe Linie zu stellen? Mußte man sich zwischen diesen Alternativen entscheiden, oder waren sie irgendwie gegeben? (Flusser 1992: 15f.)

Flusser stellt also eine gemeinsame Prager Identität in den Vordergrund, zu der die Kategorien Tscheche, Deutsche oder Jude offensichtlich nur Subkategorien bilden. Von den nationalkulturell je eindeutigen Orten in der Beschrei- 
bung Kischs her perspektiviert, wird der Prager Stadtraum so aber zu einem (fast) flächendeckenden >Zwischenraum<, den alle Prager, seien sie Deutsche, Tschechen oder Juden, als Prager teilen. Auszugliedern sind aus diesem Raum nur die eindeutig konnotierten Räume, die sich in Prag vor allem als Gebäude mit einem programmatischen nationalkulturellen Bezug zeigen: auf tschechischer Seite etwa das Nationalmuseum (Národní muzeum), das Nationaltheater (Národní divadlo) und das Gemeindehaus (Obecní dům), auf deutscher das Deutsche Theater. Allerdings wäre es, ohne dass dies hier näher auszuführen ist, unangemessenen, solche Gebäude nur als eine Manifestation des Eigenen zu verstehen; konzeptuell ist es weit angemessener, das vorsätzliche Vergessenmachen des Anderen in diesen Räumen zu fokussieren, weil so das Fremde und Andere gleichwohl noch als anwesend vorauszusetzen sind.

Eine solche Darstellung der >Tripolis Praga < als umfassender Zwischenraum entspricht im Übrigen auch neueren historischen Forschungsergebnissen, die die unzutreffende Diagnose der strikten Trennung der >Volksgruppen $<$ in Prag endlich beiseite geräumt haben (vgl. Čapková 2005). In ihrer Studie Geteilte Kulturen. Eine Geschichte der tschechisch-jüdisch-deutschen Beziehungen in Prag (1918-1938) weist Ines Koeltzsch darauf hin, dass »trotz der starken Tendenz nationaler Homogenisierung in der städtischen Gesellschaft nach dem Ersten Weltkrieg bei bi- und multilingualen Akteuren eine hohe Flexibilität im alltäglichen Umgang mit der gesprochenen und geschriebenen Sprache vorhanden war« (Koeltzsch 2012: 27). Diese sprachliche Flexibilität ist aber auch als >allgemein< kulturelle $z u$ verstehen. Auch hier also zeigt sich Identität als verflüssigt und situativ veränderbar. Gleichzeitig ist diese situative »Flexibilität im alltäglichen Umgang « gerade die Voraussetzung dafür, dass das Staunen nicht endet. Die »Juden, Deutschen, Tschechen« haben nicht abgetrennt voneinander gelebt, sondern gemeinsam Prag bevölkert; sie hatten regelmäßigen Umgang miteinander, was dazu führte, dass sie stets von Neuem in ein Staunen übereinander gerieten, dass sie - entsprechend der obigen Profilierung des interkulturellen Staunens - nicht (oder eben nicht immer) von festgefügten Bildern des Fremden ausgingen, sondern solche Bilder des nahen Fremden in stetiger Verschiebung hielten. Nur unter der Voraussetzung der strikt gegeneinander abgegrenzten Räume ließe sich ein Ende des Staunens denken, nicht aber in miteinander >geteilten Kulturen<.

Wenn jedoch die Räume in Prag wie in Kischs Darstellung eindeutig gegeneinander abgegrenzt waren (eine Diagnose, die Kisch im Übrigen auch nur deshalb so deutlich stellt, um anschließend sich selbst zum [einzigen] Überschreiter zu stilisieren, so dass auch sein Modell eine solche Grenzüberschreitung vorsieht), muss man sich fragen, ob sich vorderhand ein zur Grenze alternatives Raumkonzept (jenseits des >Zwischenraums $<$, der als Modell problematisch bleibt, weil er immer wieder als ein Raum zwischen zwei anderen verstanden werden kann) finden lässt. Dabei bietet sich das Raum-Modell des Horizonts an, der eben keinen von einer Grenze umzirkelten Raum meint. Vielmehr: »Der Horizont gehört ins semantische Feld der platonischen Chora, die Seiendem Raum gibt, ohne selbst (bloßer) Raum oder Seiendes zu sein.« (Schöning/ 
Weinberg 2004: 196) Das heißt aber nichts anderes, als dass das Raummodell des Horizonts erst einmal keine Grenzen kennt. Selbstverständlich ist innerhalb des Horizonts nicht alles eins, auch nicht alles hybrid - und so ist man innerhalb seiner, wenn man bestimmte Phänomene präzise beschreiben will, zu Grenzziehungen gezwungen. Wichtig aber ist, dass man mit dem Horizont nicht von gegeneinander gestellten und somit voneinander abgegrenzten Einheiten ausgeht. Dennoch erlaubt das Raummodell des Horizonts die Rede von Einheiten, nur muss man eben jedes Mal erklären, wie diese mehr oder weniger stabilen Einheiten zustande gekommen, wie sie hervorgebracht worden sind. Im Horizont kann man instabile Einheiten denken und nur zeitweise gültige Grenzen; man kann Vermischungen wie Verschiebungen denken und Inseln der nationalkulturellen Vereindeutigung in ihn eintragen.

Am Beispiel des Kulturraums der böhmischen Länder lässt sich leicht zeigen, dass es denkbar unangemessen wäre, das Mit- und Nebeneinander von Tschechen, Juden und Deutschen in ihm im Sinne eines Mosaiks à la Kisch einzutragen, denn das würde jeder Ethnie zwar mehrere, aber nur jeweils ihr zukommende Orte zusprechen. Im Grunde muss man - geht man vom Kulturmodell des Horizonts aus - sein >Inneres < erst einmal entleeren und kann nur vermerken, dass es von den drei >Gruppen< gemeinsam bewohnt wird. Aber bei dieser reichlich allgemeinen Aussage muss es ja nicht bleiben. Jede Art von Differenz, ob einer klaren Abgrenzung oder einem sozusagen >weichen< Miteinander geschuldet, lässt sich in ihn eintragen. Man muss nur je von Neuem rechtfertigen, warum man es auf diese Weise einträgt und somit erklärt.

Für den Kulturraum der böhmischen Länder gibt es für das Modell des Horizonts ein weiteres Argument, insofern am Anfang der Geschichtsschreibung der böhmischen Länder eben dieses Modell voraus-gesetzt wurde, nämlich in der von František Palacký verfassten ersten Geschichte von Böhmen aus der ersten Hälfte des 19. Jahrhunderts. Palacký war ein tschechischer Historiker und Politiker, der sich vehement in der tschechischen Nationalbewegung engagierte. $\mathrm{Zu}$ Anfang seiner Geschichte von Böhmen liest man:

In der Mitte des Festlandes von Europa erhebt sich, kaum tausend Quadratmeilen groß, der Schauplatz unserer Volksgeschichte, das Königreich Böhmen. Die äußeren Umrisse desselben zeichnen die Gestalt eines unregelmäßigen Vierecks, welches seine Winkel nach den vier Weltgegenden richtet. Es ist rings von Gebirgen eingeschlossen, welche auf drei Seiten zugleich die höchste Wasserscheide von Centraleuropa bilden, und erhält dadurch seine natürliche Begränzung. (Palacký 1844: 3)

Zwar ist hier von einer »Begränzung « die Rede; entscheidend aber ist, dass Palacký diese Grenzen erst einmal als natürliche Grenzen und nicht als Staatsgrenzen denkt. Was dann folgt, ist tatsächlich, Böhmen nicht von seinen Grenzen her zu denken, also abgegrenzt und von daher etwa in Differenz zu Deutschland gebracht, womit die zwei Einheiten >Deutschland < und >Böhmen < (und eine Grenze zwischen ihnen) vorausgesetzt würden, was unmittelbare Folgen auch für den Umgang mit der Geschichte Böhmens hätte. Palacký denkt die 
>äußeren Umrisse< vielmehr tatsächlich als Horizont - und Böhmen von daher als >Schauplatz<. Man hat dies in der Forschung (vgl. etwa Kořalka 2007) das >Territorialprinzip< seiner Geschichtsschreibung genannt. Palacký setzt also einen Raum, in dem die böhmische Geschichte spielt, voraus - und interessiert sich dann dafür, was sich in diesem Raum und auf diesem Schauplatz alles abgespielt hat. Mit anderen Worten, er beginnt nicht mit gegeneinander abgegrenzten Einheiten, sondern bei einer grundsätzlichen Vielfalt. So liest man weiterhin: »Die Natur bildete solchergestalt Böhmen zu einem geschlossenen Ganzen, und bedingt dadurch schon vorhinein [sic!] die Hauptzüge der böhmischen Geschichte.« (Palacký 1844: 4) Auch wenn hier wieder von einem »geschlossenen Ganzen« die Rede ist, geht es dabei nicht um eine Begrenzung gegen Andere, sondern um die Konstituierung des einen Raums - entsprechend des hier vorausgesetzten Modells des Horizonts -, in dem die böhmische Geschichte statthatte und statthat. Wie Palacký dann mit diesem Raum oder Schauplatz umgeht, zeigt sehr deutlich die folgende Passage aus der Vorrede zur ersten Auflage:

Vorzüglich möge es Kennern gefallen, das letzte Capitel des zweiten Buches aufmerksamer zu lesen. Die eigenthümlichen Schwierigkeiten einer Darstellung des alten Volkslebens der Böhmen rühren zunächst von der Verschiedenheit der Elemente her, welche sich darin abspiegeln: des allgemein slawischen, das ursprünglich vorherrschte, des deutschen, das vorzüglich seit dem X Jahrhunderte immer größeren Eingang fand, und endlich eines besonderen böhmischen, das sich zum Theil aus der Vermischung der beiden ersten erzeugte. (Ebd.: VIII [Hervorh. im Orig. gesperrt])

Palacký kennt also genuine Slawen, genuine Deutsche, aber auch Böhmen, die aus deren Mischung resultierten. Genauer noch: Er kennt slawische und deutsche und böhmische »Elemente« - und interessiert sich dafür, was mit diesen »Elementen« im Laufe der böhmischen Geschichte geschehen ist.

Um es am Ende noch einmal allgemeiner zu formulieren: Wer, wie Wolfgang Welsch, von der Hybridität ausgeht, wird irgendwann nicht darum herum kommen, in diese auch Einheiten und damit Grenzen einzutragen. Wer beim Modell der Grenze startet, wird ebenfalls bald an Grenzen des Beschreibbaren gelangen. Denn kulturelle Phänomene sind niemals eindeutig und somit klar abgrenzbar. Ein Konzept der Interkulturalität, das bei den Grenzen als Kulturmodell stehen bliebe, geriete also rasch an die Grenzen angemessener Beschreibungen. Noch einmal anders: Wer nur Grenzen kennt, wird bald an Grenzen der Beschreibung stoßen; wer nur Hybrides voraussetzt, wird bald nichts mehr präzise beschreiben können und muss zur Präzisierung seiner Beschreibungen Grenzen einfügen. Dennoch liegt es nahe, vom Modell des Horizonts - sozusagen als Raum der das Projekt der Interkulturalität prägenden Aushandlungsprozesse resp. als Ort der benannten Verschiebung - auszugehen, weil mit ihm weniger Vorentscheidungen voraus-gesetzt sind, die später dann mühsam eskamotiert werden müssen. 
So erscheint der Horizont als jenes, das dem oben als >liminales Phänomen< benannten Staunen erst einen Raum gibt, in dem es statthat. Das Staunen und die von ihm immer wieder initiierte Verschiebung beziehen sich zwar auf Grenzen, aber auf notwendig verflüssigte. Der Horizont zeigt sich so auch als angemessenes Raumkonzept des Projekts der Interkulturalität, das auf einer solchen Verschiebung >beruht<, die als Prozess nicht nur die räumliche Verschiebung von Grenzen meint (die immer im Bereich des Gewussten verbliebe), sondern auch die Anerkenntnis des Nichtwissens, das sich nicht im Bezug auf fixe Grenzen etablieren kann, sondern wiederum nur im vom Horizont eingeräumten Raum.

Im Falle der böhmischen Länder (und aller ehemals von einer spezifischen Interkulturalität geprägten Räume) hat dies noch einen besonderen Effekt: Man muss solche Interkulturalität nicht mehr als strikt vergangene denken. Im offenen Zukunftshorizont des Projekts der Interkulturalität ist nie ausgeschlossen, dass irgendwann wieder an die den Raum in früheren Zeiten prägende Interkulturalität angeschlossen werden kann. Eine einmal gelebte Interkulturalität denkt man sie als Prozess oder eben als Projekt - kann nicht wirklich beendet werden (etwa durch Vertreibung der Anderen), sondern sie ist auch danach noch da im >anderen Zustand < des derzeit nicht Aktualisierten, gleichsam als »Gegen-Gedächtnis« (Foucault 1987: 85). Man wird, da sie nur in die Vergessenheit verschoben wurde, auf sie zurückkommen können.

\section{ScHLUSS}

Als verschollen gilt jemand, der der Kunde der Menschen entzogen ist, »indem man seit langem nichts von ihm gehört hat und daher sein fortleben und sein aufenthaltsort unbekannt sind.« (Deutsches Wörterbuch 1984: Bd. 25, 1138) In Kafkas Der Verschollene ist Amerika eine entstehende und vergehende Hyperbel Europas oder, wie es in einer an Jacques Derridas L'autre cap (1990) ausgerichteten Lektüre heißen könnte, das andere (westliche) Kap Europas. Als europäische Schöpfung ähnelt es der Alten Welt, auf der anderen Seite aber entfernt es sich von ihr und bereitet dadurch den Boden, auf dem etwas Neues entsteht. Amerika ist anders und nicht anders, für Karl Roßmann vertraut und unvertraut zugleich. Unvertraut ist Amerika für Karl deswegen, weil sich die »ungeheuere Welt« (Kafka 2002b: 562 ) nicht auf den Begriff bringen lässt. »Das grenzt ja ans Wunderbare (Kafka 2008: 55), sagt Karl, als der Onkel von der schier unglaublichen Entwicklung seiner Geschäfte erzählt. Das Wunder kennt keinen Gegenbegriff (vgl. Barck 2005: 74). Wer es bezeichnen will, trägt sich mit Absichten, von denen Stephen Greenblatt in Wunderbare Besitztümer (1994) berichtet. Karl dagegen vergeht über Amerika gewissermaßen Hören und Sagen. Er staunt nur noch - selbst am vorläufigen Ende, in Oklahama, das lediglich bei Arthur Holitscher (vgl. Holitscher 1912: 367), aber ansonsten auf keiner Weltkarte zu finden ist und das in Kafkas Text als »noch größer [...], als er nur irgendwie hatte denken können« (Kafka 2008: 296), benannt wird. So ist noch Oklahama 
Sinnbild eines interkulturellen Wissens, das als verschoben ausgewiesen wird. Es macht die »Erstaunensentfernung« (Guzzoni 2012: 36) rückgängig und setzt das Nichtwissen als einen Modus der Freiheit ins Recht.

\section{LITERATUR}

Alt, Peter-André (2008): Franz Kafka. Der ewige Sohn. Eine Biographie. 2. Aufl. München.

Aristoteles (1995): Philosophische Schriften. Bd. 5: Metaphysik. Nach der Übersetzung v. Hermann Bonitz bearb. v. Horst Seidl. Hamburg.

Barck, Karlheinz (2005): Art. "Wunderbar». In: Ders. u.a. (Hg.): Ästhetische Grundbegriffe. Historisches Wörterbuch in sieben Bänden. Bd. 6. Stuttgart/Weimar, S. $730-773$.

Beck, Ulrich (1996): Wissen oder Nicht-Wissen? Zwei Perspektiven "reflexiver Modernisierung". In: Ders./Anthony Giddens/Scott Lash: Reflexive Modernisierung. Eine Kontroverse. Frankfurt a.M., S. 289-316.

Brandstetter, Gabriele (1999): Fremde Zeichen. Zu Gottfried Kellers Novelle ,Die Berlocken. Literaturwissenschaft als Kulturpoetik. In: Jahrbuch der deutschen Schillergesellschaft 43, S. 305-324.

Brod, Max (1918): Ein menschlich-politisches Bekenntnis. Juden, Deutsche, Tschechen. In: Die neue Rundschau 29, Bd. 2, S. 1580-1593.

Čapková, Kateřína (2005): Češi, Němci, Židé? Národní identita Židů v Čechách. Prag.

Dies. (2013): Czechs, Germans, Jews? National Identity and the Jews of Bohemia. New York [engl. Übers. v. Čapková 2005).

Dembeck, Till (2010): X oder U? Herders Interkulturalitätı. In: Dieter Heimböckel u.a. (Hg.): Zwischen Provokation und Usurpation. Interkulturalität als (un)vollendetes Projekt der Literatur- und Sprachwissenschaften. München, S. 103-128.

Derrida, Jacques (1990): L'autre cap. Paris.

Deutsches Wörterbuch (1984). Von Jacob und Wilhelm Grimm. 32 Bde. [Bd. 1-16 in 32 TIn.]. Leipzig 1854-1954. Erg.-Bd.: Quellenverzeichnis, ebd. 1971 - Nachdruck 33 Bde. München.

Dietsche, Petra (1984): Das Erstaunen über das Fremde. Vier literaturwissenschaftliche Studien zum Problem des Verstehens und der Darstellung fremder Kulturen. Frankfurt a.M.

Eisner, Pavel (1948): Franz Kafka a Praha. In: Kritický měsíčník 9, H. 1-2, S. 66-82.

Flusser, Vilém (1992): Bodenlos. Eine philosophische Autobiographie. Mit einem Nachw. v. Milton Vargas. Bensheim.

Földes, Csaba (2009): Black Box Interkulturalitätı. Die unbekannte Bekannte (nicht nur) für Deutsch als Fremd-/Zweitsprache. Rückblick, Kontext und Ausblick. In: Wirkendes Wort 59, S. 503-525.

Fornet-Betancourt, Raúl (2007): Interkulturalität in der Auseinandersetzung. Frankfurt a.M./London.

Foucault, Michel (1987): Nietzsche, die Genealogie, die Historie. In: Ders.: Von der Subversion des Wissens. Frankfurt a.M., S. 69-90. 
Gaier, Ulrich (2007): Herder als Begründer des modernen Kulturbegriffs. In: Germanisch-Romanische Monatsschrift 57, H. 1, S. 5-18.

Goethe, Johann Wolfgang von (1985): Italienische Reise. Sonderausgabe. Hg. und komm. v. Herbert von Einem. München.

Greenblatt, Stephen (1994): Wunderbare Besitztümer. Die Erfindung des Fremden: Reisende und Entdecker. Aus dem Engl. v. Robin Cackett. Berlin.

Gugerli, David/Sarasin, Philipp (2009): Editorial [zum Themenheft Nicht-Wissen]. In: Nach Feierabend. Zürcher Jahrbuch für Wissensgeschichte 5, S. 7-9.

Guzzoni, Ute (2012): erstaunlich und fremd. Erfahrungen und Reflexionen. Freiburg/ München.

Habermas, Jürgen (1981): Kleine politische Schriften I-IV. Frankfurt a.M., S. 444-464.

Heidegger, Martin (1980): Die Zeit des Weltbilds. In: Ders.: Holzwege. 6., durchges. Aufl. Frankfurt a.M., S. 73-110.

Heimböckel, Dieter (2013a): Die deutsch-französischen Beziehungen aus interkultureller Perspektive. In: Zeitschrift für interkulturelle Germanistik 4, H. 2, S. 19-39.

Ders. (2013b): Wie vom Zufall geführt. Kleists Griffel. In: Ders. (Hg.): Kleist. Vom Schreiben in der Moderne. Bielefeld, S. 23-46.

Heinrich, Klaus: (2000): Vom Bündnis denken. Religionsphilosophie. Basel/Frankfurt a.M.

Hermsdorf, Klaus (1961): Kafka, Weltbild und Roman. Berlin.

Hess-Lüttich, Ernest W.B. (2003): Interkulturelle Kommunikation. In: Alois Wierlacher/ Andrea Bogner (Hg.): Handbuch interkulturelle Germanistik. Stuttgart/Weimar, S. $75-81$.

Holitscher, Arthur (1912): Amerika heute und morgen. Reiseerlebnisse. Berlin.

James, Henry (1949): The American. Introduction by Joseph Warren Beach. New York u.a.

Kafka, Franz (2002a): Kritische Ausgabe. Der Verschollene. Apparatband. Hg. v. Jost Schillemeit. Frankfurt a.M.

Ders. (2002b): Kritische Ausgabe. Tagebücher. Hg. v. Hans-Gerd Koch, Michael Müller u. Malcolm Pasley. Frankfurt a.M.

Ders. (2008): Der Verschollene. Roman. In der Fassung der Handschrift. Frankfurt a.M. Kimmich, Dorothee/Schahadat, Schamma (2012): Einleitung. In: Dies. (Hg.): Kulturen in Bewegung. Beiträge zur Theorie und Praxis der Transkulturalität. Bielefeld, S. 7-21.

Kisch, Egon Erwin (1990): Deutsche und Tschechen. In: Ders.: Marktplatz der Sensationen. Entdeckungen in Mexiko. Hg. v. Bodo Uhse u. Gisela Kisch. 2. Aufl. Berlin, S. 75-83.

Kluge, Alexander (2006): „Projekte sind im Grunde Vorgriffe, Ausbrüche in die Ferne“. Alexander Kluge im Gespräch mit Claus Philipp. In: Christian Reder (Hg.): Lesebuch Projekte. Vorgriffe, Ausbrüche in die Ferne. Wien/New York, S. 9-29.

Koeltzsch, Ines (2012): Geteilte Kulturen. Eine Geschichte der tschechisch-jüdischdeutschen Beziehungen in Prag (1918-1938). München.

Kořalka, Jiři (2007): František Palacký (1798-1876). Der Historiker der Tschechen im österreichischen Vielvölkerstaat. Wien.

Kramer, Fritz (1977): Verkehrte Welten. Zur imaginären Ethnographie des 19. Jahrhunderts. Frankfurt a.M. 
Langenohl, Andreas/Poole, Ralph/Weinberg, Manfred (Hg.; 2015): Transkulturalität. Klassische Texte. Bielefeld [im Druck].

Lazardzig, Jan (2007): Theatermaschine und Festungsbau. Paradoxien der Wissensproduktion im 17. Jahrhundert. Berlin.

Lichtenberg, Georg Christoph: Schriften und Briefe. Bd. 2: Sudelbücher II: Materialhefte, Tagebücher. Hg. v. Wolfgang Promies. Darmstadt.

Lindner, Frank (2010): Projektwissensmanagement. Status quo, Gestaltungsfaktoren und Erfolgsdeterminanten des Wissensmanagements in der Projektabwicklung. Münster.

Martens, Ekkehard (2003): Vom Staunen oder Die Rückkehr der Neugier. Leipzig.

Mecklenburg, Norbert (2008): Das Mädchen aus der Fremde. Germanistik und interkulturelle Literaturwissenschaft. München.

Montaigne, Michel de (1953): Essais. Auswahl und Übers. v. Herbert Lüthy. Zürich.

Nancy, Jean-Luc (1993): Lob der Vermischung. In: Lettre International, H. 21, S. 6 f.

Nicolai; Ralf R. (1981): Kafkas Amerika-Roman "Der Verschollene". Motive und Gestalten. Würzburg.

Palacký, Franz (1844): Geschichte von Böhmen. Größtentheils nach Urkunden und Handschriften. Der ersten Auflage zweiter Abdruck. 1. Bd.: Die Urgeschichte und die Zeit der Herzöge in Böhmen bis zum Jahr 1197. Prag.

Rieger, Stefan/Schahadat, Schamma/Weinberg, Manfred (1999): Interkulturalität zwischen Inszenierung und Archiv. Vorwort. In: Dies. (Hg.): Interkulturalität. Zwischen Inszenierung und Archiv. Tübingen, S. 9-26.

Schlesier, Renate (1996): Das Staunen ist der Anfang der Anthropologie. In: Hartmut Böhme/Klaus R. Scherpe (Hg.): Literatur und Kulturwissenschaften. Positionen, Theorien, Modelle. Reinbek b. Hamburg, S. 47-59.

Schmitz, Walter (1991): Das Eigene als das Fremde: Interkulturalitätı als Forschungsparadigma für die ,Germanistikı. In: Bernd Thum/Gonthier-Louis Fink (Hg.): Praxis interkultureller Germanistik. Forschung - Bildung - Politik. Stuttgart, S. 171-175.

Schnell, Ralf (Hg.; 2000): Metzler Lexikon Kultur der Gegenwart. Stuttgart/Weimar.

Schütz, Alfred (1972): Der Fremde. Ein sozialpsychologischer Versuch. In: Ders: Gesammelte Aufsätze II. Studien zur soziologischen Theorie. Hg. v. Arvid Brodersen. Den Haag, S. 53-69.

Schnyder, Mireille (2013): Überlegungen zu einer Poetik des Staunens im Mittelalter. In: Martin Baisch/Andreas Degen/Jana Lüdtke (Hg.): Wie gebannt. Ästhetische Verfahren der affektiven Bindung von Aufmerksamkeit. Freiburg i. Br., S. 95-114.

Schöning, Matthias/Weinberg, Manfred (2004): Ironie der Grenzen - Horizonte der Interkulturalität. In: Aleida Assmann/Ulrich Gaier/Gisela Trommsdorff (Hg.): Positionen der Kulturanthropologie. Frankfurt a.M., S. 196-222.

Seel, Martin (2009): Vom Nachteil und Nutzen des Nicht-Wissens für das Leben. In: Nach Feierabend. Zürcher Jahrbuch für Wissensgeschichte 5, S. 37-49.

Stanitzek, Georg (1987): Der Projektmacher. Projektionen auf eine ,unmöglicher moderne Kategorie. In: Ästhetik \& Kommunikation 17, H. 65/66, S. 135-146.

Timm, Uwe (1997): Das Nahe, das Ferne. Schreiben über fremde Welten. In: Paul Michael Lützeler (Hg.): Deutsche Schriftsteller berichten aus der Dritten Welt. Frankfurt a.M., S. 34-48.

Tugendhat, Ernst (2004): Egozentrizität und Mystik. Eine anthropologische Studie. 2. Aufl. München. 
Waldenfels, Bernhard (1999): Topographie des Fremden. Studien zur Phänomenologie des Fremden 1. 2. Aufl. Frankfurt a.M.

Ders. (2000): Zwischen den Kulturen. In: Jahrbuch Deutsch als Fremdsprache 26, S. $245-261$.

Weinberg, Manfred (2014): Heimat in der Prager deutschen Literatur und bei Franz Kafka. In: Carme Bescansa/Ilse Nagelschmidt (Hg.): Heimat als Chance und Herausforderung. Repräsentationen der verlorenen Heimat. Berlin, S. 155-176.

Welsch, Wolfgang (2009): Was ist eigentlich Transkulturalität? In: Lucyna Darowska/ Claudia Machold (Hg.): Hochschule als transkultureller Raum? Beiträge zur Kultur, Bildung und Differenz. Bielefeld, S. 39-66.

Ders. (2012): Was ist eigentlich Transkulturalität? In: Dorothee Kimmich/Schamma Schahadat (Hg.): Kulturen in Bewegung. Beiträge zur Theorie und Praxis der Transkulturalität. Bielefeld, S. 25-40.

Wierlacher, Alois (2003): Interkulturelle Germanistik. Zu ihrer Geschichte und Theorie. Mit einer Forschungsbibliographie. In: Ders./Andrea Bogner (Hg.): Handbuch interkulturelle Germanistik. Stuttgart/Weimar, S. 1-45.

Willke, Helmut (2002): Dystopia. Studien zu einer Krisis des Wissens in der modernen Gesellschaft. Frankfurt a.M.

Yousefi, Hamid Reza/Braun, Ina (2011): Interkulturalität. Eine interdisziplinäre Einführung. Darmstadt. 